\title{
MAN.07 - Hazard Analysis and Critical Control Points (HACCP) in the production of yellow fever IFA (Active Pharmaceutical Ingredient).
}

\author{
Carla Cristina Alves Bustamante Rosa ${ }^{1 *}$; Miguel Angel de la O Herrera ${ }^{1}$; Elezer Monte Blanco \\ Lemes ${ }^{1}$.
}

1Fiocruz/Bio-Manguinhos.

Introduction: The global trend of the pharmaceutical industry is to use the concept of risk analysis to provide a considered increase in safety and ensure the quality of production of immunobiologicals, becoming an important tool in the incessant search for continuous improvement. The HACCP (Hazard Analysis and Critical Control Point) tool assists in this purpose because of its ability to identify critical process points based on prior knowledge and proposing control and monitoring actions in key process parameters.

Objective: The aim of this work is to identify critical control points through the HACCP risk analysis tool in the production stages of the IFA (active pharmaceutical input) of an attenuated virus vaccine.Through this identification improvement actions will be implemented to optimize and increase the reliability of the production process, aiming at the first actions for the product space design.

Methodology: First the risk analysis techniques were applied: Hazard Analysis and Critical Control Points - HACCP and for the classification and prioritization of the hazards evidenced and the Risk Matrix tool. Subsequently, the productive processes were evaluated and the potential hazards were identified and analyzed according to their degree of severity and frequency. Finally, they were submitted to the application of the 7 principles of the HACCP tool, where the critical points of control and elaboration of the corrective actions methodologies of monitoring and verification of the CCPs were defined.

Results: 157 hazards were raised in the productive stages listed in this work: $57.9 \%$ biological, $40.7 \%$ physical and $1.27 \%$ chemical. Of this total, only $19 \%$ were considered CCPs and were monitored. It was essential then to intensify the potential of the tool and to monitor the CCPs, to define control measures for all identified hazards, and to map all existing and proposed control measures. The measures were evaluated for their effectiveness in eliminating and/or reducing the risks to acceptable levels, without losing the safety and quality of the processes performed.The critical limits for each CCP were defined according to historical data and recent studies available. Thus, a plan of action was developed for all the risks raised and defined monitoring and verification proposals according to the periodicity of the routine of the productive processes.

Conclusion: Pharmaceutical industries are looking for technological alternatives capable of subsidizing recent regulatory changes. And that should be sufficient to reduce and/or eliminate the risks inherent in the steps, without directly interfering in production yields. Understanding all the factors involved in the risks and directing the identified control strategies becomes imminent being essential for the protection of the product. This makes it possible to ensure greater efficiency in exposure, reduction and mitigation of risks.

Keywords: HACCP; Yellow fever; CCP 